\title{
Application of DQE for quantitative assessment of detectors to estimate AEC efficiency in digital mammography
}

\author{
Ewa FABISZEWSKA ${ }^{\mathrm{a},{ }^{*}}$ Anna WYSOCKA-RABIN ${ }^{\mathrm{b}}$, Magdalena DOBRZYŃSKA ${ }^{\mathrm{b}}$, Witold SKRZYŃSKI ${ }^{\mathrm{a}}$, Katarzyna \\ PASICZ $^{\mathrm{a}}$ \\ ${ }^{a}$ Medical Physics Department, Maria Sklodowska-Curie National Research Institute of Oncology (MSCNRIO), Warsaw, \\ Poland \\ ${ }^{b}$ Particle Acceleration Physics and Technology Division, National Centre for Nuclear Research (NCNR), Otwock Swierk, \\ Poland \\ "E-mail address: ewa.fabiszewska@pib-nio.pl
}

\begin{abstract}
Optimisation of the detector's exposure parameters settings for image quality and patient dose is an important task in digital mammography. Assessment of a digital detector's performance can be done objectively and without operator bias by determining the Detective Quantum Efficiency (DQE). The authors of this article aim to prove that the performance of the AEC system can be objectively portrayed through DQE. The results were examined for influence of $\mathrm{KAD}$ changes on $\mathrm{DQE}$ values and to determine if it was possible to obtain similar DQE values for different exposures. While analysing the effect of the operation of the AEC system described with DQE, the doses received by women during mammography examinations were considered, as well.

The AEC system's exposure control mechanism cannot guarantee the same DQE value for different object thicknesses. When the object thickness increases, the AEC system should increase the KAD value to obtain the same DQE value. The result of increasing KAD would be the increase of mean glandular dose for some women. However, assuming that DQE is a good indicator of image quality, introducing the proposed changes to the AEC system's operation would result in the same image quality for all breast thicknesses.

This approach to DQE use for AEC system evaluation is independent of the image processing procedure and can be the basis for changes to system calibration done by the manufacturer's technical support team.
\end{abstract}

Key words: DQE; AEC; mammography.

\section{Introduction}

It is possible to control directly the exposure of the image detector in analogue systems by evaluation of the darkening of the film plate. However, that is not the case with digital systems. The user of the device cannot influence the way the automatic exposure control (AEC) system operates. The detector calibration procedure is done by the unit service and the images are displayed in predetermined greyscale, regardless of the parameters of the patient's exposure. Images produced with incorrect exposure values can be corrected by regulating the parameters of the shown image. Exposure parameters (tube voltage and tube load) that are too low result in poorer image quality, whereas exposure parameters set too high cause the increase in the dose received by the patient. That is why optimisation of the detector's exposure parameters settings for image quality and the patient dose is an important task in digital mammography.

Assessment of a digital detector's performance can be done objectively and without operator bias by determining the Detective Quantum Efficiency (DQE). Usually, this parameter is applied in the context of comparisons of different mammography systems and radiation detectors, as well as the influence of exposure parameters onto DQE. ${ }^{1-10}$ Marshall et al. have proven the correlation between DQE and image quality, which is indirectly influenced by the automatic exposure control, ${ }^{11}$ as previously stated. DQE is dependent on the X-ray beam quality and the air kerma at detector entrance (KAD), ${ }^{1}$ which are set by the AEC.

The authors of this article aim to prove that the performance of the AEC system can be objectively portrayed through DQE. If the AEC system sets exposure in such a way that DQE values remain largely constant regardless of an object's thickness, then the hypothesis is true. This was verified by determining DQE for exposures set by the AEC system under clinical conditions. The results were examined for the influence of KAD changes on DQE values and to determine if it was possible to obtain similar DQE values for different exposures. The operation of the AEC system described by DQE was also analysed from the point of view of doses that women during mammography examinations. 


\section{Materials and Methods}

\section{Imaging system}

In our study, we used a Siemens Mammomat Inspiration digital mammography unit. The Siemens Mammomat detector has a layer of amorphous selenium and a-Si TFT matrix readout with a pixel size of $85 \mu \mathrm{m}$. The mammography unit was equipped with a molybdenum and tungsten anode with additional filtration of molybdenum and rhodium. The mammography unit system offers the options of a manual setting, semiautomatic exposure control (AEC) and full-automatic exposure control (OPDOSE).

\section{Phantom exposures}

In order to determine which exposure parameters were chosen by the AEC system, exposures of PMMA phantoms of the following thicknesses were tested: $2 \mathrm{~cm}, 3 \mathrm{~cm}, 4 \mathrm{~cm}, 4.5 \mathrm{~cm}, 5$ $\mathrm{cm}, 6 \mathrm{~cm}$ and $7 \mathrm{~cm}$. Appropriate spacers were added to each phantom thickness to achieve a thickness equivalent to that of a breast after compression per the European Guidelines. ${ }^{12}$ During the exposure, the AEC system was set to OPDOSE mode, which is used in clinical examinations. In this mode, exposure parameters (tube voltage and load) are chosen depending on the thickness of a breast after compression for only one anode/filter combination, i. e. W/Rh.

\section{DQE determination}

A computer program was created to determine DQE - it will be described in another publication. The input data for the program was comprised of images produced using an aluminium filter $2 \mathrm{~mm}$ thick placed under the tube, results of air kerma measurements using a radiation meter (Piranha Black 457, RTI Electronics AB, Sweden) and images of an edge test device. The edge test device was a $1 \mathrm{~mm}$ thick stainless-steel plate constructed specifically for this work and produced at the National Centre for Nuclear Research, with the angle of the edge equal to $2^{\circ}$. DQE was determined for different tube voltage values as a function of KAD.

All calculations in the program were in accordance with standards of the International Electrotechnical Commission (IEC 62220-1-2:2007). ${ }^{13}$ The procedure for calculating DQE was verified with MIQuaELa software. ${ }^{14}$ The relative uncertainty of the calculated DQE value $u_{D Q E}$ is less than $\pm 10 \%$ (with coverage factor $\mathrm{k}=2$ ).

\section{Average glandular dose (AGD)}

Performance of the AEC system was tested in terms of patients' exposure to $\mathrm{X}$ radiation. Average glandular dose (AGD) was determined for all phantom thicknesses (see Phantom exposures section) and for clinical exposures done using the unit that was tested. Clinical parameters of radiation exposure received by 224 women aged 43-86 were recorded over a two-week period. These exposures were set by the AEC system in the OPDOSE mode. The median age of the patients was 62 years, and the total number of exposures was 896 .

AGD values for the exposure of phantoms were determined according to the European Guidelines [12]. The AGDs received by women were determined by the Dance method [15]. The obtained AGD values were compared with the acceptable limits presented in the European Guidelines [12]. The uncertainty in determining the mean glandular dose was $14 \%$ [16]. The meter used to measure the air kerma was the same, as for the measurements performed DQE determination (see DQE determination section).

\section{Assessment of detector exposure and image quality}

The performed exposures of phantoms of various thicknesses provided information on the values of tube voltage and tube load selected by the AEC system in OPDOSE mode. For these exposures, the values of the air kerma at the detector entrance (KAD) were determined by measuring the air kerma behind the phantoms, taking into account the anti-scatter grid coefficients and converting the obtained results to source-image distance (SID).

For the above-mentioned tube voltage values, the DQE as a function of KAD was determined. In this case, KAD was measured behind a $2 \mathrm{~mm}$ thick aluminium filter and converted from the square of the distance to the SID distance. It did not require taking into account the anti-scatter grid factor. This allowed determining the $\mathrm{DQE}$ for the KAD value for the exposure of each phantom.

\section{Results and discussion}

Table 1 shows exposure parameters for different phantom thicknesses, done with the AEC system set in OPDOSE mode, which is used during clinical examinations. The table also contains KAD and AGD values that correspond to individual phantom exposures. KAD values ranged from $63 \mu \mathrm{Gy}$ (for the $7 \mathrm{~cm}$ phantom exposure) to $108 \mu \mathrm{Gy}$ (for the $2 \mathrm{~cm}$ phantom exposure). Marshall et al. previously analysed the quantum noise value to examine the image detector exposure. ${ }^{2}$ They established the ranges of KAD values for different mammography systems, for which image quality is determined mainly by quantum noise. The range of KAD values for the Siemens Mammomat Inspiration unit is between $22 \mu \mathrm{Gy}$ and $390 \mu \mathrm{Gy} .^{2}$ When compared with the table, it is apparent that the AEC system in OPDOSE mode applied KAD for each phantom thickness in order to prevent electronic noise from limiting image quality.

Acceptable limits for AGD values for each phantom thickness according to the European Guidelines ${ }^{12}$ are also presented in the table. AGD values determined for the phantom exposures are nearly two times lower than the specified limits, which suggests that the AEC system worked properly in this regard. 
Table 1. Exposure parameters in OPDOSE mode.

\begin{tabular}{|c|c|c|c|c|c|c|c|}
\hline Phantom thickness [cm] & 2.0 & 3.0 & 4.0 & 4.5 & 5.0 & 6.0 & 7.0 \\
\hline Equivalent breast thickness after compression [cm] & 2.1 & 3.2 & 4.5 & 5.3 & 6.0 & 7.5 & 9.0 \\
\hline Anode/filter & $\mathrm{W} / \mathrm{Rh}$ & W/Rh & $\mathrm{W} / \mathrm{Rh}$ & W/Rh & $\mathrm{W} / \mathrm{Rh}$ & $\mathrm{W} / \mathrm{Rh}$ & $\mathrm{W} / \mathrm{Rh}$ \\
\hline Tube voltage $[\mathrm{kV}]$ & 26 & 27 & 28 & 29 & 30 & 31 & 32 \\
\hline Tube load [mAs] & 43.6 & 65.2 & 97 & 109.1 & 123.8 & 174.7 & 236.8 \\
\hline $\mathrm{KAD}[\mu \mathrm{Gy}]$ & 108.73 & 95.77 & 89.57 & 82.70 & 78.38 & 70.77 & 63.54 \\
\hline AGD [mGy] & 0.48 & 0.65 & 0.91 & 1.07 & 1.24 & 1.70 & 2.20 \\
\hline Acceptable AGD limit [mGy] & 1.0 & 1.5 & 2.0 & 2.5 & 3.0 & 4.5 & 6.5 \\
\hline
\end{tabular}

The DQE values were determined for tube voltage that corresponded to the ones selected in OPDOSE mode for exposures of different phantom thicknesses and for the following tube load values: $2.5 \mathrm{mAs}, 6.3 \mathrm{mAs}, 12.5 \mathrm{mAs}, 25.0$ $\mathrm{mAs}, 50.0 \mathrm{mAs}$ and $100.0 \mathrm{mAs}$. This made it possible to estimate the changes in DQE as a function of KAD and for different tube voltages. Table 2 shows KAD values for different tube voltages and loads.

Changes in DQE in relation to $\mathrm{KAD}$ for five spatial resolution values (1 $\mathrm{lp} / \mathrm{mm}$ to $5 \mathrm{lp} / \mathrm{mm})$ are shown in Figure 1 for $26,29.32 \mathrm{kV}$ (respectively, starting from the top). These figures show DQE as a function of KAD - in the direction parallel to the anode-cathode axis. Points on the graphs were connected to illustrate the relations between the values. The range of KAD on the horizontal axis was limited to $300 \mu \mathrm{Gy}$, since the phantom exposures in clinical examinations did not exceed that level. The vertical line on each graph shows the exposure value chosen in OPDOSE mode. The presented figures correspond only to the $2 \mathrm{~cm}, 4.5 \mathrm{~cm}$ and $7 \mathrm{~cm}$ phantom exposures. For the remaining thicknesses of the phantoms, analogous relationships were obtained and they were not included in Figure 1.

The DQE value increases along with air kerma at detector entrance for all spatial frequencies, which is in line with the observations by Marshall et al. ${ }^{11}$ The increase in DQE and $\mathrm{KAD}$ varies depending on the tube voltage. The slant of the DQE curve shows that as KAD increased, the growth of DQE slowed. The DQE curve never reached a plateau.

Table 3 shows the KAD and DQE values in both directions corresponding to the exposures of all phantom thicknesses. As the tube voltage increases, KAD decreases for each phantom exposure. One could expect that KAD values in exposures made with the AEC system would stay similar regardless of phantom thickness. However, the KAD value for $32 \mathrm{kV}$ behind a phantom of $7 \mathrm{~cm}$ was nearly two times lower than the KAD value for $26 \mathrm{kV}$ behind a $2 \mathrm{~cm}$ phantom.

Table 3. KAD and DQEx and DQEy values corresponding to phantom exposures for $5 \mathrm{lp} / \mathrm{mm}$.

\begin{tabular}{cccccccc}
\hline $\begin{array}{c}\text { Phantom } \\
\text { thickness }[\mathrm{cm}]\end{array}$ & $\mathbf{2 . 0}$ & $\mathbf{3 . 0}$ & $\mathbf{4 . 0}$ & $\mathbf{4 . 5}$ & $\mathbf{5 . 0}$ & $\mathbf{6 . 0}$ & $\mathbf{7 . 0}$ \\
\hline $\mathrm{KAD}[\mu \mathrm{Gy}]$ & 108.73 & 95.77 & 89.57 & 82.70 & 78.38 & 70.77 & 63.54 \\
$\mathrm{DQEx}$ & 0.22 & 0.22 & 0.22 & 0.21 & 0.21 & 0.18 & 0.17 \\
$\mathrm{DQEy}$ & 0.21 & 0.20 & 0.20 & 0.18 & 0.18 & 0.18 & 0.18 \\
\hline \hline
\end{tabular}

Table 2. KAD values for which DQE was measured.

\begin{tabular}{|c|c|c|c|c|c|c|c|}
\hline \multirow{2}{*}{\multicolumn{2}{|c|}{ KAD $[\mu \mathbf{G y}]$}} & \multicolumn{6}{|c|}{ Tube load [mAs] } \\
\hline & & 2.5 & 6.3 & 12.5 & 25 & 50 & 100 \\
\hline \multirow{7}{*}{ 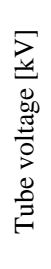 } & 26 & 5.6 & 15.9 & 32.7 & 66.5 & 134.1 & 269.2 \\
\hline & 27 & 6.8 & 19.0 & 38.8 & 78.7 & 158.7 & 318.6 \\
\hline & 28 & 7.6 & 21.7 & 44.5 & 90.5 & 182.5 & 366.6 \\
\hline & 29 & 8.3 & 24.1 & 49.9 & 101.9 & 205.9 & 414.0 \\
\hline & 30 & 9.6 & 27.3 & 56.2 & 114.5 & 231.0 & 463.9 \\
\hline & 31 & 10.8 & 30.4 & 62.3 & 126.8 & 255.7 & 513.3 \\
\hline & 32 & 11.6 & 33.1 & 68.0 & 138.5 & 279.4 & 561.3 \\
\hline
\end{tabular}

$26 \mathrm{kV}$
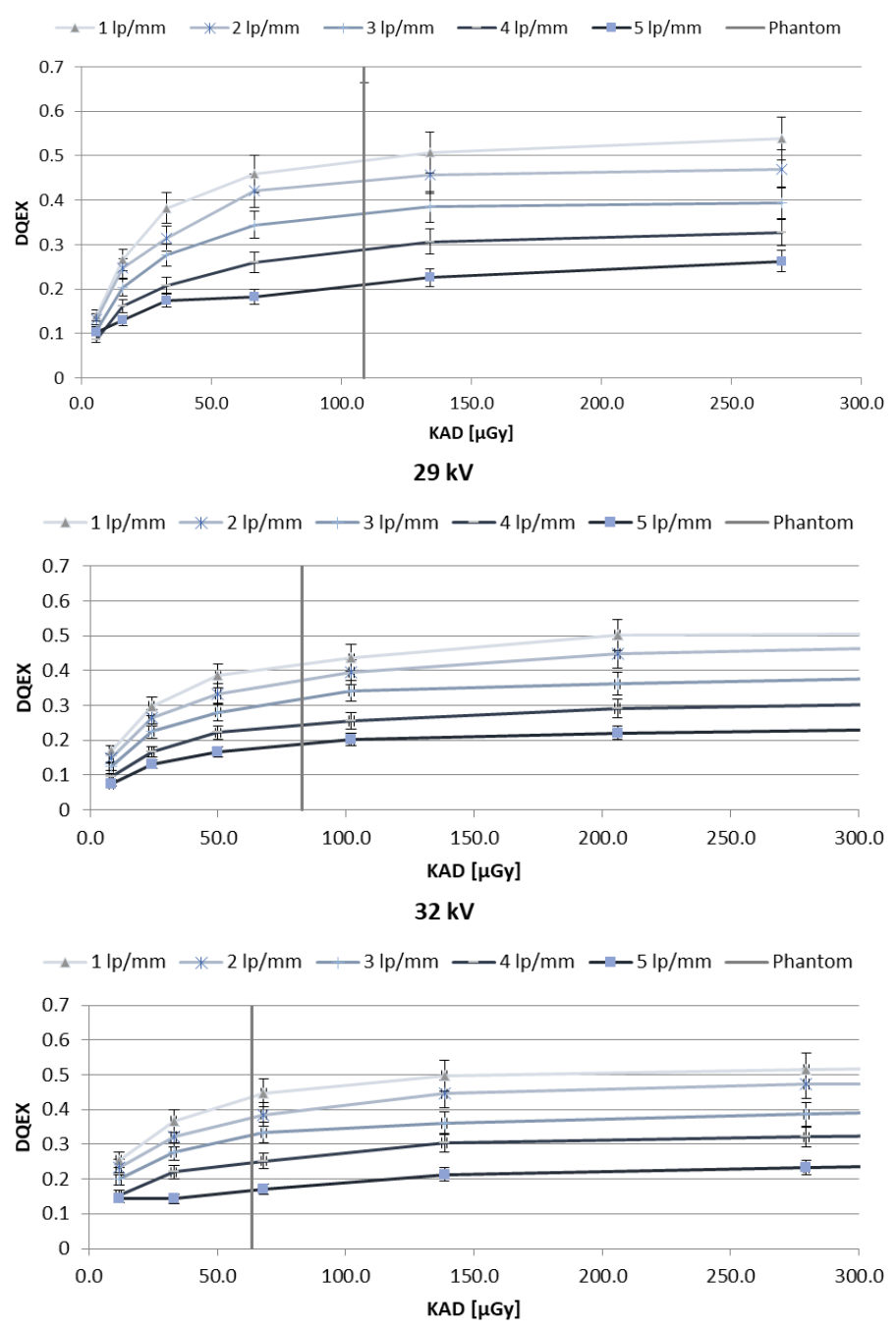

Figure 1. DQEX and DQEY as a function of KAD for five different spatial frequencies: graph pairs a-b, c-d, e-f, and g-h are for the following tube voltages: $26 \mathrm{kV}, 29 \mathrm{kV}$ and $32 \mathrm{kV}$ respectively, for the $\mathrm{W} / \mathrm{Rh}$ anode/filter combination. 
That meant that the DQE value for $5 \mathrm{lp} / \mathrm{mm}$ decreased by about $23 \%$ in the $\mathrm{X}$ direction and about $14 \%$ in the $\mathrm{Y}$ direction. This was the greatest gap between DQE values determined for the different phantoms. Therefore, lower KAD values for exposures of $6 \mathrm{~cm}$ phantoms or thicker can be expected to yield lower object visibility in comparison to exposures taken with thinner phantoms.

After comparing the DQE values as a function of KAD for each phantom thickness, a change to the AEC system was proposed to optimize DQE values, i.e. similar to the values for $5 \mathrm{lp} / \mathrm{mm}$ for a $2 \mathrm{~cm}$ phantom. For example, to achieve optimal DQE for images of $6 \mathrm{~cm}$ phantoms or thicker, it would be necessary to increase exposure to a level where KAD would equal about $130 \mu \mathrm{Gy}$. That would roughly double AGD for an equivalent breast thickness of $7.5 \mathrm{~cm}$ after compression, which would have affected $4 \%$ of the women whose cases were examined for this analysis (Figure 3).

Figure 2 shows the distribution of breast thickness after compression for 224 women. While Figure 3 shows exposition parameters chosen by OPDOSE mode during examinations. The tube voltage ranged from $24 \mathrm{kV}$ to $34 \mathrm{kV}$. The most often chosen tube voltage was $30 \mathrm{kV}$ (Figure 3a). The minimum

(a)

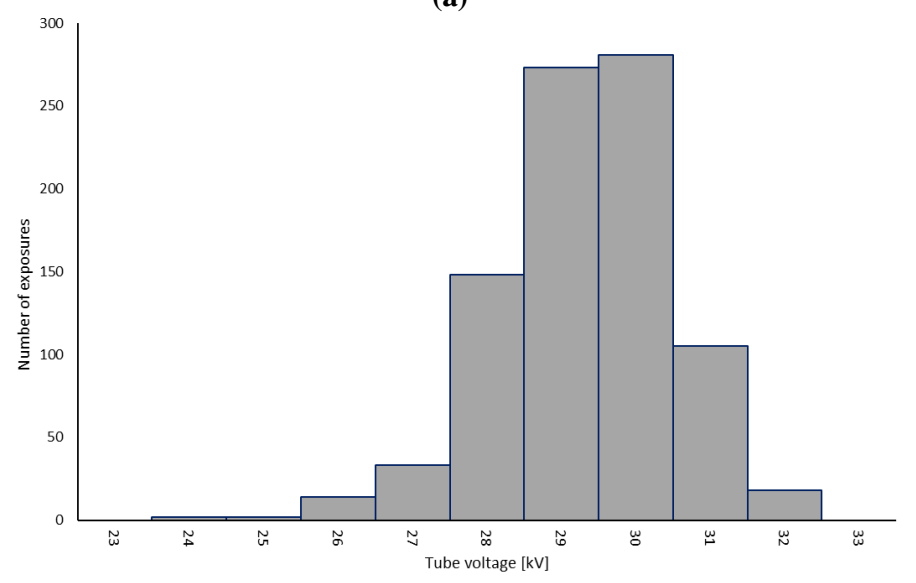

tube load value was $39 \mathrm{mAs}$ and the maximum tube load value was $353 \mathrm{mAs}$, with a median value of $113 \mathrm{mAs}$ (Figure 3b). Figure 4a is a histogram of individual doses received by women using the unit that was tested. AGD median was $1.15 \mathrm{mGy}$, while the maximum value was $3.69 \mathrm{mGy}$. For all exposures, AGD remained within the acceptable limit recommended in the European Guidelines.

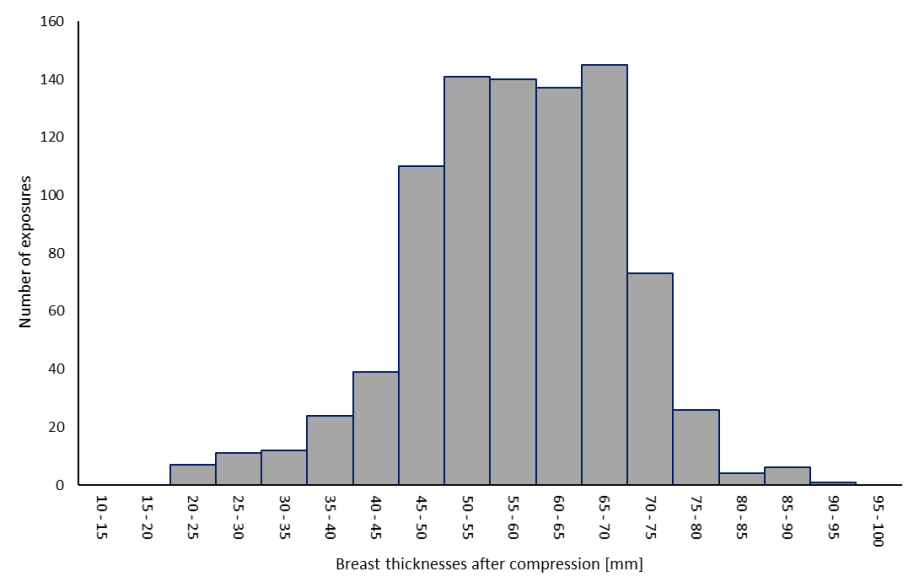

Figure 2. A histogram of breast thicknesses after compression for 224 women.

(b)

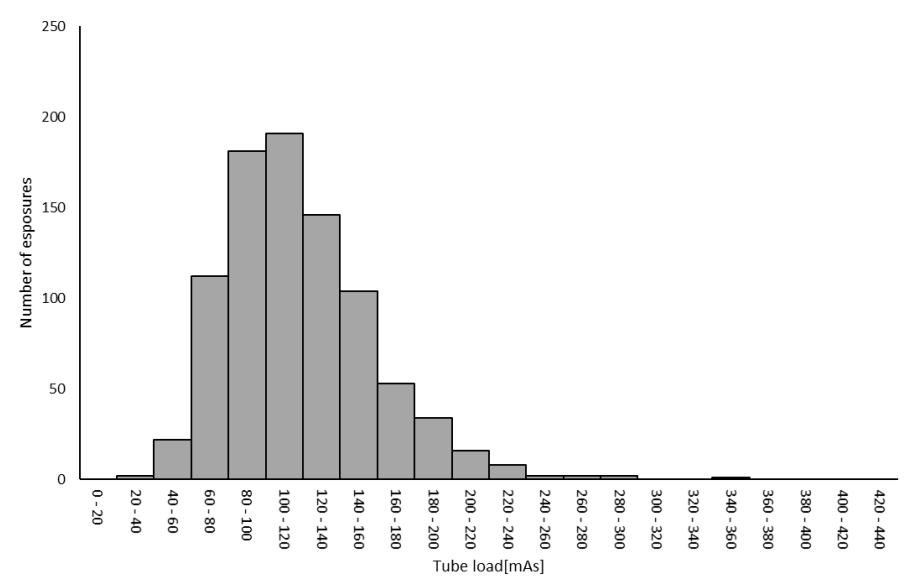

Figure 3. A histogram of (a) tube voltage value and (b) tube load for 896 exposures taken during medical examinations of 224 women.

(a)

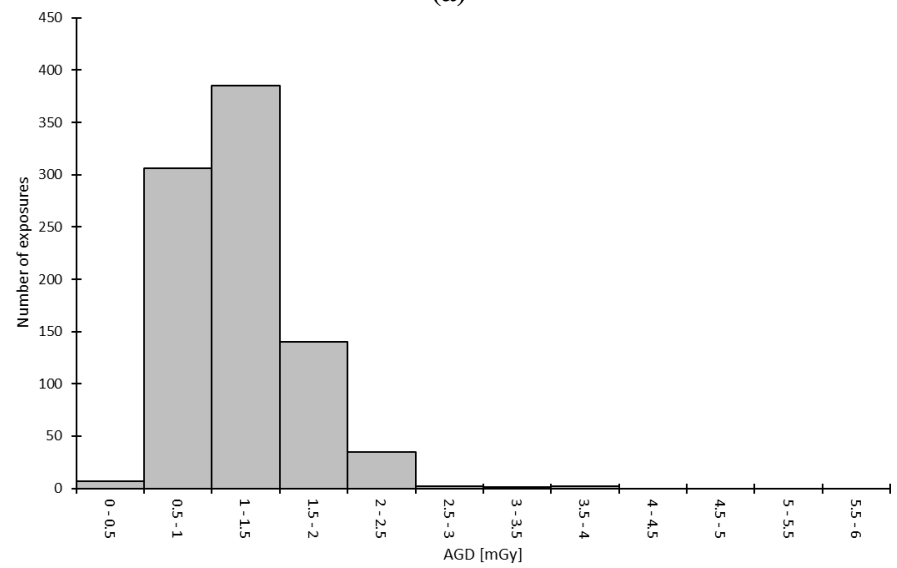

(b)

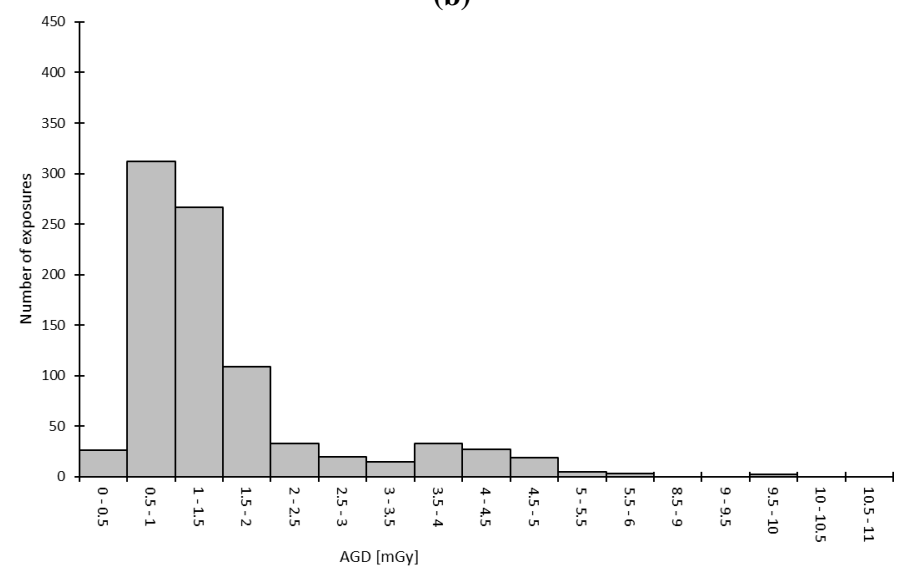

Figure 4. A histogram of AGD for routine clinical conditions (a) and for equalised DQE (b). 
Figure $4 \mathbf{b}$ shows what the distribution of doses would be after increasing the exposure to achieve similar DQE. The median would remain $1.15 \mathrm{mGy}$, and the maximum value would be $9.92 \mathrm{mGy}$. The European Guidelines would be met for $95 \%$ of exposures.

When the AEC system is applied in OPDOSE mode under clinical conditions, DQE values vary greatly for exposures of objects of different thicknesses. The largest difference was $20 \%$. The relation of DQE as a function of KAD shows that DQE grows much slower at KAD above $70 \mu \mathrm{Gy}$. The observed correlation can be used to set an exposure level that will result in similar DQE values for all object thicknesses. However, introducing the proposed changes in clinical practice would result in some women receiving increased doses.

Linking AEC system operation to DQE will allow the user to choose an option that could be expected to deliver the best image quality. A smaller increase in exposure could be considered. This exposure would not raise DQE as much but would not lead to a significant increase in AGD for women. In the future, the authors of this study plan to extend the program to include the quality of the X-ray spectrum and phantom scattering as it is proposed in the works on the determination of eDQE. ${ }^{17,18}$

\section{Conclusions}

It has been observed that the AEC system's exposure control mechanism cannot guarantee the same DQE value for different object thicknesses. When the object thickness increases, the AEC system should increase the KAD value to obtain the same DQE value. The result of increasing KAD would be the increase of mean glandular dose for some women. However, assuming that DQE is a good indicator of image quality, introducing the proposed changes to the AEC system's operation would result in the same image quality for all breast thicknesses.

\section{References}

1. García-Mollá R, Linares R, Ayala R. Study of DQE dependence with beam quality on GE essential mammography flat panel. J Appl Clin Med Phys. 2010;12(1):3176. https://doi.org/10.1120/jacmp.v12i1.3176

2. Marshall NW, Monnin P, Bosmans H, Bochud FO, Verdun FR. Image quality assessment in digital mammography: part I. Technical characterization of the systems. Phys Med Biol. 2011;56(14):4201-4220. https://doi.org/10.1088/0031-9155/56/14/002

3. Gershan V, Ristova MM, Ivanovski Z. Evaluation of the Hologic Selenia FFDMsystem with tungsten tube. In: The First International Conference on Radiation and Dosimetry in Various Field of research, RAD 2012 Proceedings. 2012

4. Monnin P, Bosmans H, Verdun FR, Marshall N. A comprehensive model for quantum noise characterization in digital mammography. Phys Med Biol. 2016;61:2083-2108. https://doi.org/10.1088/0031-9155/61/5/2083

5. Oberhofer N, Fracchetti A, Springeth M, Ehrenfried M. Digital mammography--DQE versus optimized image quality in clinical environment: an on site study. Proceedings Volume 7622, Medical Imaging 2010: Physics of Medical Imaging. https://doi.org/10.1117/12.844150

6. Ghetti C, Borrini A, Ortenzia O, Rossi R, Ordóñez, PL. Physical characteristics of GE Senographe Essential and DS digital mammography detectors. Med Phys. 2008:35:456-463. https://doi.org/10.1118/1.2828185

7. NHS Breast Screening Programme Equipment Report. Technical evaluation of GE Healthcare Senographe Pristina digital breast tomosynthesis system. 2019. Available: https://www.gov.uk/government/publications/breast-screening-ge-senographe-pristina-dbtsystem-evaluation

8. NHS Breast Screening Programme Equipment Report. Technical evaluation of Siemens Mammomat Inspiration digital breast tomosynthesis system - modified detector and software (VB60). 2018. Available: https://www.gov.uk/government/publications/breast-screening-siemens-mammomat-inspiration-vb60

9. NHS Breast Screening Programme Equipment Report. Technical Evaluation of Hologic 3Dimensions digital mammography system in 2D mode. 2019. Available: https://www.gov.uk/government/publications/breast-screening-hologic-3d-digital-mammographysystem-in-2d-mode

10. Technical evaluation of Siemens Inspiration PRIME with VB30L software NHS Breast Screening Programme Equipment Report 1503. 2016. Available: https://www.gov.uk/government/publications/breast-screening-evaluation-of-siemens-inspiration-prime

11. Marshall NW. A comparison between objective and subjective image quality measurements for a full field digital mammography system. Phys Med Biol. 2006;51:2441-63. https://doi.org/10.1088/0031-9155/51/10/006

12. European Commission. European Guidelines for Quality Assurance in Breast Cancer Screening and Diagnosis. Fourth edition, supplements, 2013.

13. International Electrotechnical Commission. Medical electrical equipment - Characteristics of digital X-ray imaging devices - Part 1-2: Determination of the detective quantum efficiency - Detectors used in mammography. IEC 62220-1-2:2007 
14. Ayala R, Linares R, García-Mollá R. MIQuaELa, software for DQE measuring in digital radiography/mammography. In: Dössel O., Schlegel W.C. (eds) World Congress on Medical Physics and Biomedical Engineering, September 7 - 12, 2009, Munich, Germany. IFMBE Proceedings, vol 25/2. Springer, Berlin, Heidelberg. https://doi.org/10.1007/978-3-642-03879-2_230

15. Dance DR, Skinner CL, Young KC, Beckett JR, Kotre CJ. Additional factors for the estimation of mean glandular breast dose using the UK mammography dosimetry protocol. Phys Med Biol. 2000;45:3225-40. https://doi.org/10.1088/0031-9155/45/11/308

16. Chevalier M, Morán P, Ten JI, et al. Patient dose in digital mammography. Med Phys. 2004;31:2471-2479. https://doi.org/10.1118/1.1784591

17. Salvagnini E, Bosmans H, Struelens L, Marshall NW. Effective detective quantum efficiency for two mammography systems: Measurement and comparison against established metrics. Med Phys. 2013;40:101916. https://doi.org/10.1118/1.4820362

18. Salvagnini E, Bosmans H, Struelens L, Marshall N. Effective detective quantum efficiency (eDQE) and effective noise equivalent quanta (eNEQ) for system optimization purposes in digital mammography. Progress in Biomedical Optics and Imaging - Proceedings of SPIE. 8313. https://doi.org/10.1117/12.911193 